\title{
Alteración de la fertilidad masculina por hiperplasia suprarrenal congénita. Azoospermia reversible con terapia de glucocorticoide
}

\author{
ENZO DEVOTO C. ${ }^{1}$, MARCIA MADARIAGA A..$^{2, a}$, WANDA FERNÁNDEZ ${ }^{3}$
}

'Sección Endocrinología, Servicio de Medicina, Hospital Clínico San Borja Arriarán, Departamento de Medicina, Facultad de Medicina, Campus Centro, Universidad de Chile. ${ }^{2}$ Instituto de

Investigaciones Materno Infantil, Facultad de Medicina, Campus Centro, Universidad de Chile. Santiago de Chile. ${ }^{3}$ Servicio de Anatomía Patológica, Hospital Clínico San Borja Arriarán. aTecnólogo Médico.

Recibido el 13 de agosto de 2009, aceptado el 7 de julio de 2011.

Correspondencia a: Dr. Enzo Devoto C. Hernando de Aguirre 194 Of. 62 Fax: 2290238 Santiago, Chile E-mail: edevoto@vtr.net

\section{Congenital adrenal hyperplasia causing male infertility. Report of one case}

In males, congenital adrenal hyperplasia due to 21 hydroxylase deficiency is associated to normal fertility or infertility caused by a hypogonadotrophic hypogonadism $(\mathrm{HH})$ or gonadal damage caused by intratesticular adrenal remnants. We report a 29-year-old male with azoospermia, without any important personal or family background. Physical examination was normal, his height was $150 \mathrm{~cm}$ and his testicular volume was $10 \mathrm{ml}$ (normal 15 to $25 \mathrm{ml}$ ). Laboratory showed a normal testosterone and FSH and LH in the low normal limit. These results discarded a $\mathrm{HH}$, whose diagnostic requirements are a low testosterone and inadequately normal or low gonadotrophins. A testicular biopsy was informed as compatible with HH. A 21 hydroxylase deficiency was suspected and confirmed with extremely high levels of 17 hydroxyprogesterone at baseline and after stimulation with fast acting ACTH. Clomiphene citrate did not increase testosterone or gonatrophin levels. Testicular ultrasound discarded the presence of adrenal nodules. Betametasone therapy resulted in a normal testicular development, normalization of sperm count, reduction of 17 hydroxyprogesterone and testosterone levels with an ulterior rise of the latter. Spontaneous paternity was achieved twice. It must be remembered that in cases of azoospermia due to congenital adrenal hyperplasia, testosterone produced by adrenal glands hinders the laboratory diagnosis of $\mathrm{HH}$.

(Rev Med Chile 2011; 139: 1060-1065).

Key words: Adrenal hyperplasia, congenital; Hypogonadism; Infertility, male.
L

a corteza suprarrenal estimulada por ACTH sintetiza cortisol, andrógenos (androstenediona, dehidroepiandrosterona (DHA), dehidroepiandrosterona-sulfato (DHA-S)) y aldosterona, que es regulada además por angiotensina II y potasio.

En la esteroidogénesis suprarrenal diversas enzimas actúan sobre precursores obteniendo como producto final dichas hormonas. Las enzimas son codificadas por genes cuya mutación altera la síntesis y actividad de la enzima; siendo las más comunes el déficit de 21 hidroxilasa (90\%) y de 11 beta hidroxilasa.
La deficiencia severa de 21 hidroxilasa (CYP21) impide la conversión de progesterona en 11 desoxicorticosterona y de la 17 hidroxiprogesterona en 11 desoxicortisol, acumulándose los precursores progesterona y 17 hidroxiprogesterona, disminuyendo cortisol y/o aldosterona (Figura 1).

La hipocortisolemia aumenta la secreción de CRH hipotalámico y ACTH hipofisiario produciéndose hiperplasia de la corteza adrenal y estimulándose el crecimiento de restos adrenales presentes en el testículo desde la etapa embrionaria (RTGS).

El déficit enzimático aumenta androstene- 


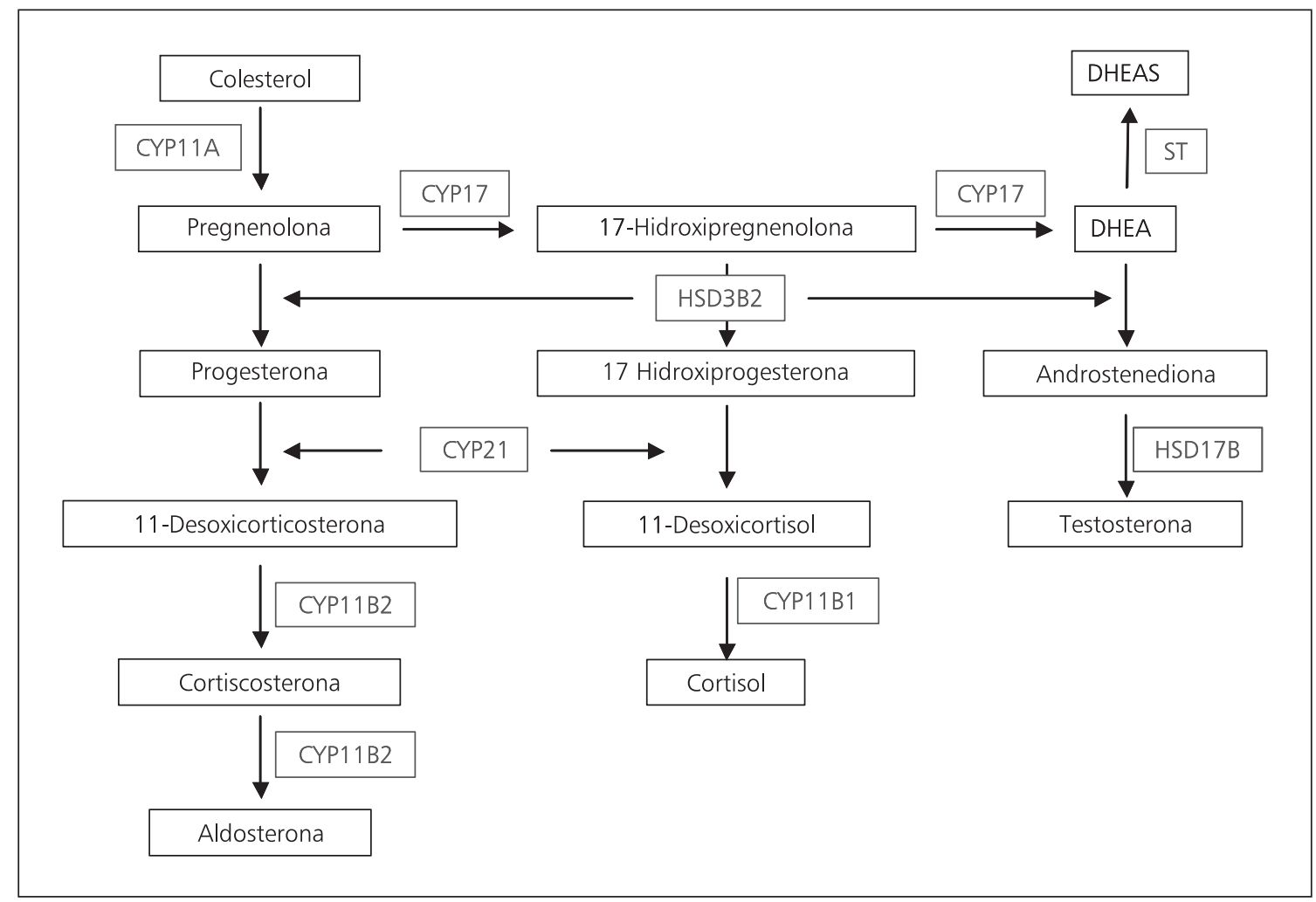

Figura 1. Representación esquemática de la esteroidogénesis.

diona y testosterona ( $\mathrm{T}$ ) que en la forma severa intrauterina (hiperplasia suprarenal congénita clásica), viriliza los genitales externos femeninos y en el varón puede aumentar el tamaño peneano (macrogenitosomía).

En la hiperplasia suprarrenal congénita ( HSC) clásica, el $25 \%$ presenta la forma virilizante pura, acelerándose crecimiento lineal y maduración ósea con posterior detención y talla baja; aumento de masa muscular y en el varón pubarquia sin incremento de tamaño testicular. La forma perdedora de sal (75\% de HSC) presenta severa morbilidad y mortalidad al no diagnosticarse precozmente el déficit de cortisol y aldosterona ${ }^{1}$.

En el varón con defecto menos severo del gen (HSC no clásica o de inicio tardío) se produce infertilidad o sospecha de tumor testicular por crecimiento de RTGS².

Los nódulos generados por hiperplasia de RTGS reemplazan al parénquima testicular y/o comprimen los ductos eferentes impidiendo el paso de espermatozoides. Son más frecuentes en la forma perdedora de sal y alterarían desde la infancia las células de Leydig y Sertoli ${ }^{3}$.

Wilkins demostró en HSC clásica el daño gonadal con biopsias que indicaban ausencia de células de Leydig, túbulos seminíferos con sólo espermatogonias y/o células de Sertoli; tratando con cortisona aumenta el volumen testicular y logra desarrollo leydigiano y túbulos con espermatogénesis activa ${ }^{4}$.

La HSC clásica por déficit de la 21 hidroxilasa cursa con 17 hidroxiprogesterona basal elevada y post estímulo con ACTH, androstenediona elevada, $T$ elevada en el prepuber y normal en el post puberal, derivada de la interconversión periférica de la androstenediona suprarrenal.

La fertilidad se altera por hipogonadismo hipogonadotropo $(\mathrm{HH})$ secundario a la alteración hormonal de la HSC o daño testicular por crecimiento de RTGS .

La terapia con glucocorticoide restaura fertilidad en HSC con HH y en algunos con RTGS ${ }^{5-12}$.

Se han comunicado casos de pacientes no 
tratados o que abandonando terapia presentan espermiograma y fertilidad normal ${ }^{13,14}$, posiblemente por ausencia de RTGS o menor sensibilidad a la frenación hipotalámica.

Presentamos un caso de infertilidad masculina debida a HSC que, tratado con glucocorticoides , normalizó función gonadal.

\section{Caso clínico}

Varón de 29 años que consultó por infertilidad primaria documentándose azoospermia.

Sin antecedentes familiares ni personales, asintomático y vida sexual normal.

Examen físico: 1,50 m, $63 \mathrm{~kg}$, presión arterial 120- $80 \mathrm{mmHg}$, sin ginecomastia, bien androgenizado y gran desarrollo muscular.

Examen genital: pene normal, vello pubiano $\mathrm{V}$ de Tanner, testículos de consistencia normal, volumen de $10 \mathrm{ml}$ (orquidómetro de Prader, $\mathrm{VN}=15-25 \mathrm{ml}$ ), epidídimo, deferente y próstata normal; varicocele (-).

Laboratorio: 2 espermiogramas con volumen de $2 \mathrm{ml}, \mathrm{pH} 8$, ausencia de espermatozoides al examen directo y post centrifugación confirmaron azoospermia.

Testosterona $3,8 \mathrm{ng} / \mathrm{ml}(\mathrm{V} \mathrm{N}=2-6 \mathrm{ng} / \mathrm{ml})$, LH 2,0 mUI / ml ( V N = 0,4-5,7) FSH 2,0 mUI/ $\mathrm{ml}(\mathrm{V} \mathrm{N}=2-15)$

Ante una azoospermia normotestosterónica, con LH normal y sin alza de FSH, se sospechó azoospermia obstructiva indicándose biopsia testicular.

Informe histopatológico: Túbulos disminuidos de calibre con membrana basal gruesa y en algunas áreas fibrótica; epitelio tubular compuesto casi exclusivamente por células de Sertoli, en algunas zonas células germinales escasas que no alcanzan maduración completa. Ausencia de células de Leydig (Figura 2).

El diagnóstico histopatológico no es concordante con la hipótesis diagnóstica por lo cual se reevaluó el caso.

Se planteó HSC por fenotipo: talla baja, acentuado desarrollo muscular, testículos disminuidos de tamaño, valores de testosterona normal y gonadotropinas en límite normal bajo.
Reinterrogado el paciente, no refería historia de vómitos, diarreas u hospitalización sugerente de déficit de mineralo y glucocorticoide.

Se diagnosticó HSC clásica por elevación de 17 hidroxiprogesterona basal y post estímulo con ACTH (Tabla 1).

La administración de citrato de clomifeno (100 mg/día por 10 días) demostró descenso de gonadotropinas y ausencia de elevación de la T compatible con $\mathrm{HH}$. La respuesta normal es alza de $100 \%$ de la $\mathrm{T}$ y aumento de gonadotropinas.

No se dispuso de actividad de renina (una actividad de renina elevada, sugeriría déficit de mineralocorticoide), androstenediona, ni estudio de mutaciones en el gen de la 21 hidroxilasa.
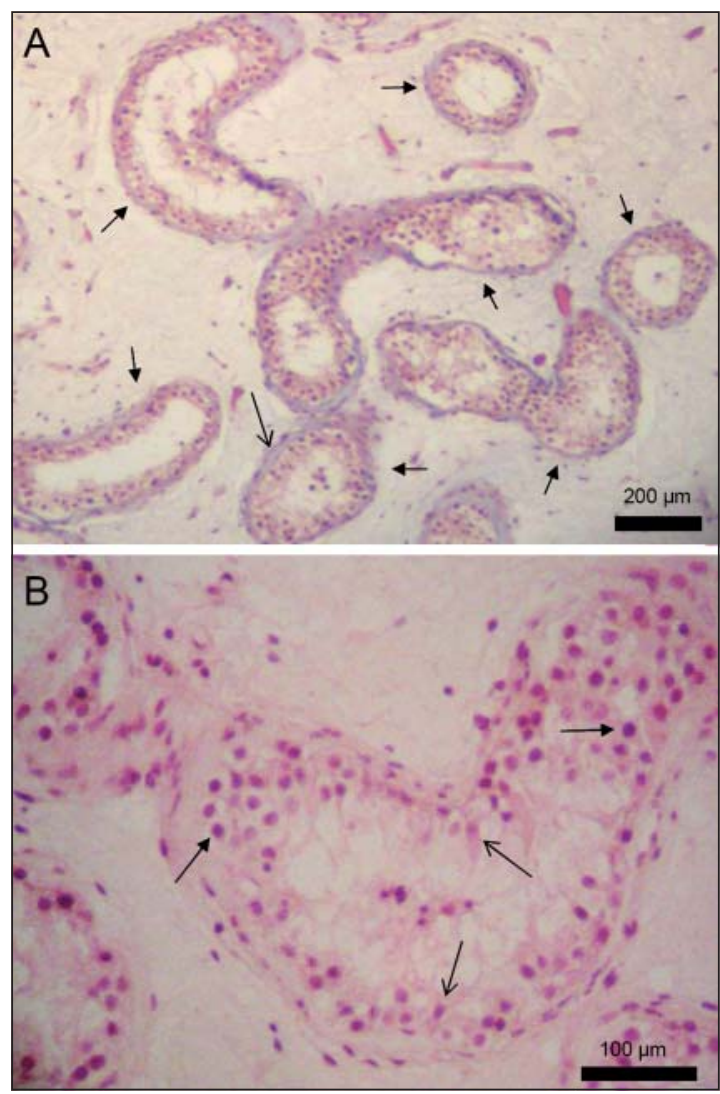

Figura 2. Panel A: Biopsia testicular donde se observa túbulos seminíferos (flechas cerradas) con engrosamiento de la membrana basal (flecha abierta), intersticio ensanchado y ausencia de células de Leydig. Tinción Mason. Panel B: A mayor aumento, túbulos semíniferos casi exclusivamente con células de Sertoli (flechas abiertas) y muy escasas células germinales (flechas cerradas). Tinción Hematoxilina- eosina. 
Tabla 1. Test de ACTH rápido

\begin{tabular}{|lcccc|}
\hline Test de ACTH (rápido) & Basal & Valor normal & $\mathbf{6 0}$ minutos & Valor normal post ACTH \\
\hline 17 hidroxiprogesterona $(\mathrm{ng} / \mathrm{ml})$ & 152 & $0,55-1,33$ & 225 & $<10$ \\
Cortisol $(\mathrm{ug} / \mathrm{ml})$ & 12,7 & $5-25$ & 19,5 & $>18$ \\
\hline
\end{tabular}

Tabla 2. Respuesta a la terapia con betamatasona

\begin{tabular}{|c|c|c|c|c|}
\hline Tratamiento & $\begin{array}{c}\text { Previo } \\
\text { tratamiento }\end{array}$ & $\begin{array}{l}90 \text { días post } \\
\text { tratamiento }\end{array}$ & $\begin{array}{l}240 \text { días post } \\
\text { tratamiento }\end{array}$ & $\begin{array}{c}\text { Valores } \\
\text { normales }\end{array}$ \\
\hline $17 \mathrm{OH}$ progesterona (ng/ml) & 152 & 1,77 & 4,0 & $0,55-1,33$ \\
\hline Testosterona (ng/ml) & 3 & 1,8 & 2,65 & $2,0-6,0$ \\
\hline $\mathrm{FSH}(\mathrm{mUl} / \mathrm{ml})$ & 2,0 & & 5,4 & $4,0-20$ \\
\hline LH (mUl/ml) & 3,0 & & 11 & $4,0-20$ \\
\hline Volumen testicular (ml) & 10 & 12 & 20 & 20 \\
\hline Concent. Esperm (millón/ml) & Azoospermia & 800.000 & 20.800 .000 & $>20.000 .000$ \\
\hline Motilidad progresiva & - & $47 \%$ & $75 \%$ & $>50 \%$ \\
\hline Morfología ( OMS) & - & $62 \%$ & $59 \%$ & $>35 \%$ \\
\hline
\end{tabular}

Ecotomografía testicular : testículos de tamaño disminuido, no se demuestra existencia de nódulos intratesticulares.

\section{Conclusión diagnóstica}

Azoospermia de causa pre-testicular por hipogonadismo hipogonadotropo secundario a HSC por déficit de la 21 hidroxilasa, forma virilizante pura.

\section{Terapia y evolución}

Betametasona, $0,6 \mathrm{mg}$ a las 23 horas. La Tabla 2 muestra la evolución de los niveles hormonales, espermiograma y desarrollo testicular, pre terapia y a los 90 y 240 días de terapia.

Destacamos disminución de 17 hidroxiprogesterona, caída inicial de testosterona seguida de un alza al rango normal, normalización de gonadotropinas, volumen testicular y espermiograma.

Bajo terapia se logró paternidad espontáneamente en dos oportunidades con recién nacidos normales.

\section{Discusión}

El factor endocrino es causa poco frecuente de infertilidad masculina ( 0,9 a 8,9\%), en nuestra experiencia $3,9 \%{ }^{15}$.

La literatura generalmente no menciona a la
HSC dentro de estas causas y no aparece en casuísticas importantes ${ }^{16-18}$.

El caso clínico demostró la dificultad de diagnosticar $\mathrm{HH}$ por déficit de la 21 hidroxilasa, debido a que el algoritmo de estudio de $\mathrm{HH}$ exige $\mathrm{T}$ baja con LH baja o inadecuadamente normal para el valor disminuido de $\mathrm{T}^{16}$. En el $\mathrm{HH}$ por HSC la T es normal por su origen suprarrenal, lo que impide diagnosticar $\mathrm{HH}$ de acuerdo al algoritmo señalado.

$\mathrm{Al}$ inicio del estudio, $\mathrm{T}$ y gonadotropinas normales descartaron $\mathrm{HH}$, hipogonadismo hipergonadotropo ( $\mathrm{T}$ normal o baja con FSH y LH aumentadas) y falla tubular aislada ( $\mathrm{T}$ y $\mathrm{LH}$ normal y FSH aumentada).

Se sospechó azoospermia obstructiva indicándose biopsia testicular.

La histología reveló severo daño tubular y ausencia de células de Leydig, compatible con $\mathrm{HH}$, descartando causa obstructiva en que la histología es normal.

El fenotipo y la historia clínica hizo sospechar la HSC confirmada por el estudio de laboratorio, no disponiéndose de estudio genético.

No conocer el genotipo CYP21, no impide el diagnóstico ni la decisión terapéutica, pero es importante para diagnóstico prenatal, consejería genética ${ }^{1} \mathrm{y}$ conocer prevalencia de mutaciones.

La semiología testicular y fundamentalmente 
la ecotomografía descartan la existencia de RTGS que se presentan como áreas hipoecogénicas de aspecto nodular ${ }^{19}$.

En HH funcional, es importante conocer la respuesta al clomifeno del eje hipotálamo hipófisis testicular, para diagnóstico y terapia ${ }^{7,20}$.

El antiestrógeno citrato de clomifeno suprime la inhibición hipotalámica del GnRH por el estradiol aumentando gonadotropinas y T, en este caso la disminución de las gonadotropinas es compatible con $\mathrm{HH}$ no modificándose la $\mathrm{T}$ debido a su origen extratesticular no LH dependiente.

En la HSC los elevados niveles de progesterona, 17 hidroxiprogesterona y andrógenos suprarrenales directamente o aromatizados a estrógeno, frenan el Gn RH lo que hace disminuir la estimulación testicular por FSH y LH.

La terapia con glucocorticoide suprime CRH y ACTH disminuyendo andrógenos y gestágenos adrenales y su acción frenadora hipotalámica, recuperándose el eje hipotálamo hipofisiario permitiendo a, FSH desarrollar el túbulo seminífero y a LH diferenciar células de Leydig, produciéndose T intratesticular que en conjunto con FSH normalizan la espermatogénesis.

El aumento de volumen testicular, la normalización del espermiograma y ascenso de la T, luego de la caída de T de origen suprarrenal, demostraron la recuperación total del eje hipotálamo hipófisis gonadal.

El caso clínico nos alerta sobre la imposibilidad de diagnosticar HH por HSC en el varón infértil utilizando la definición clásica de $\mathrm{HH}$.

Para evitar el subdiagnóstico de esta causa endocrina de infertilidad, recomendamos solicitar 17 hidroxiprogesterona basal y post ACTH ante:

1. Sospecha clínica de HSC clásica por anamnesis y examen físico.

2. Sospecha de HSC no clásica ante severo compromiso espermático sin causa diagnosticable, con $\mathrm{T}$ y gonadotropinas normales.

Este caso concuerda con publicaciones que señalan la reversión de la azoospermia con glucocorticoides, debiendo considerarse la HSC, especialmente la forma con $\mathrm{HH}$ sin RTGS, una infertilidad de origen endocrinológico tratable.

\section{Referencias}

1. Speiser PW. Congenital adrenal hyperplasia owing to 21-hydroxylase deficiency. En: Speiser P W, ed. Endocri- nology and Metabolism Clinics of North America 2001; 30: 31-59.

2. Mussig K, Kaltenbach S, Maser-Gluth C, Hartmann MF, Wudy SA, Horger M, et al. Late diagnosis of congenital adrenal hyperplasia due to 21-hydroxylase deficiency. Exp Clin Endocrinol Diabetes 2006; 114: 208-14.

3. Martínez-Aguayo A, Rocha A, Rojas N, García C, Parra $\mathrm{R}$, Lagos $\mathrm{M}$, et al. Testicular adrenal rest tumors and Leydig and Sertoli cell function in boys with classical congenital adrenal hyperplasia. J Clin Endocrinol Metab 2007; 92: 4583-9.

4. Wilkins L, Cara J. Further studies on the treatment of congenital adrenal hyperplasia with cortisone. J Clin Endocr 1954; 14: 287-96.

5. Murphy H, George C, De Kretser D, Judd S. Successful treatment with ICSI of infertility caused by azoospermia associated with adrenal rest in the testes. Hum Rep 2001; 16: 263-7.

6. Cutfield R, Bateman JM, Odell W. Infertility caused by bilateral testicular masses secondary to congenital adrenal hyperplasia (21-hydroxylase deficiency). Fertil Steril 1983; 40: 809-14.

7. Bonaccors IA, Adler I, Figueiredo J. Male infertility due to congenital adrenal hyperplasia: testicular biopsy findings, hormonal evaluation, and therapeutic results in three patients. Fertil Steril 1987; 47: 664-70.

8. Augarten A, Weissenberg R, Pariente C, Sack J. Reversible male infertility in late onset congenital adrenal hyperplasia. J Endocrinol Invest 1991; 14: 237-40.

9. Valentino R, Savastano S, Tommaselli AP, Scarpitta MT, Dorato M, Gigante M, et al. Success of glucocorticoid replacement therapy on fertility in two adult males with 21-CAH homozygote classic form. J Endocrinol Invest 1997; 20: 690-4.

10. Nicopoullos JD, Ramsay JW, Cassar J. From zero to one hundred million in six months : the treatment of azoospermia in congenital adrenal hyperplasia. Arch Androl 2003; 49: 257-63.

11. Yang RM, Fefferman RA, Shapiro CE. Reversible infertility in a man with 21-hydroxylase deficiency congenital adrenal hyperplasia. Fertil Steril 2005; 83: 223-5.

12. Claahsen-Van Der Grinten HL, Otten BJ, Sweep FC, Hermus AR. Repeated successful induction of fertility after replacing hydrocortisone with dexamethasone in a patient with congenital adrenal hyperplasia and testicular adrenal rest tumors. Fertil Steril 2007; 88: 705.e5-705. e8.

13. Prader A, Zachmann M, Illig R. Fertility in adult males with congenital adrenal hyperplasia due to 21-hydroxylase deficiency. Acta Endocr (Kbh) 1973; Suppl 177: 57. 
14. Urban M, Lee P, Migeon C. Adult Height and Fertility in Men with Congenital Virilizing Adrenal Hyperplasia. N Engl J Med 1978; 299: 1392-96.

15. Devoto E, Madariaga M, Lioi X. Factores causales de infertilidad masculina. Contribución del factor endocrino. Rev Med Chile 2000; 128: 184-92.

16. Swerdloff R and Boyers S. Evaluation of the Male Partner of an Infertile Couple. JAMA 1982; 247: 2418-22.

17. Jarrow J. Diagnostic Approach to the Infertile Male Patient. Endocrinol Metab Clin N Am 2007; 36: 297-311.

18. Behre HM, Yeung CH, Nieschlag E. Diagnosis of Male
Infertility and Hypogonadism. En: Nieschlag E and Behre H, eds. Andrology Male Reproductive Health and Dysfunction. Berlin : Ed. Springer, 1997; 87-111.

19. Claahsen-Van Der Grinten HL, Otten BJ, Hermus AR, Sweep FC, Hulsbergen-Van De Kaa CA. Testicular adrenal rest tumors in patients with congenital adrenal hyperplasia can cause severe testicular damage. Fertil Steril 2008; 89: 597-601.

20. Devoto E, Aravena L. Hipogonadismo Hipogonadotropo funcional hipotalámico idiopático pospuberal en el varón. Rev Int Androl 2008; 6: 89-96. 